\title{
MÉTODO DA HISTERESE POR AUTO-AJUSTE PARA MINIMIZAÇÃO DE ESFORÇO DE CONTROLE NO SEPARADOR SUBMARINO VASPS
}

\author{
André V. de Melo* \\ andreedep.fem.unicamp.br \\ José R. P. Mendes* \\ jricardo@dep.fem.unicamp.br \\ *UNICAMP/FEM/DEP - C.P. 6122 - CEP 13081-970 - Campinas (SP) \\ ${ }^{\dagger}$ UNESP /IGCE/DEMAC- C.P. 178 - CEP 13506-900 - Rio Claro (SP)
}

Adriane B. S. Serapião ${ }^{\dagger}$

adrianedrc.unesp.br

\section{RESUMO}

Neste trabalho apresenta-se o estudo do desenvolvimento de um controlador não-tradicional baseado em um mecanismo de histerese com auto-ajuste para o controle de nível de líquido de um sistema de separação e bombeio submarino conhecido como VASPS. O controlador desenvolvido gera sinais enviados para a bomba centrífuga submersa para controlar o nível de líquido no tanque do separador, evitando que ele atinja valores muito baixos que poderiam danificar a bomba ou valores muito altos que reduziriam a eficiência da separação líquido/gás. Os sinais de controle gerados pelo controlador visam solicitar a bomba o mínimo possível de modo a evitar o seu desgaste e falhas prematuras. Nas simulações, o controlador desenvolvido foi testado sob grandes variações nas condições de operação, tais como golfadas, produzindo resultados bastante satisfatórios e promissores.

PALAVRAS-CHAVE: separação submarina, BCS, engenharia de petróleo, controle inteligente, histerese.

\section{ABSTRACT}

This work presents a study of the development of a nonstandard controller based on an auto-tuning hysteresis mechanism, in order to control the liquid level inside of a sub sea separation and pumping system. The developed controller

Artigo submetido em 17/09/2008 (Id:894)

Revisado em 14/10/2008 e em 21/10/2008

Aceito sob recomendação do Ed.Associado Prof. José Roberto C.Piqueira generates signals, which are sent to the electrical submersible pump to control the liquid level in the separator's tank to avoid that it reach very low values, which could damage the pump, or very high values, which would reduce the liquid/gas separation efficiency. The controller must generate the minimum number of signals variations as possible to avoid wear on the pump and premature failures. In computer simulations, the developed controller was tested under large variations in operation conditions, such as slugs, with reliable and promising results.

KEYWORDS: subsea separation, ESP, petroleum engineering, smart control, hysteresis.

\section{INTRODUÇÃO}

O sistema VASPS (vertical annular separation and pumping system) apresenta um conceito inovador de separação e bombeamento bifásico submarino (França et al., 1996). Ele é composto, basicamente, de um separador ciclônico submarino e uma bomba do tipo BCS (bombeio centrífugo submerso). No separador ciclônico podem-se identificar três estágios de separação:

- Separação primária: é composta por uma câmara de expansão, onde grande parte do gás é separado;

- Separação secundária: é composta por uma estrutura helicoidal e utiliza o princípio de separação centrífuga; 


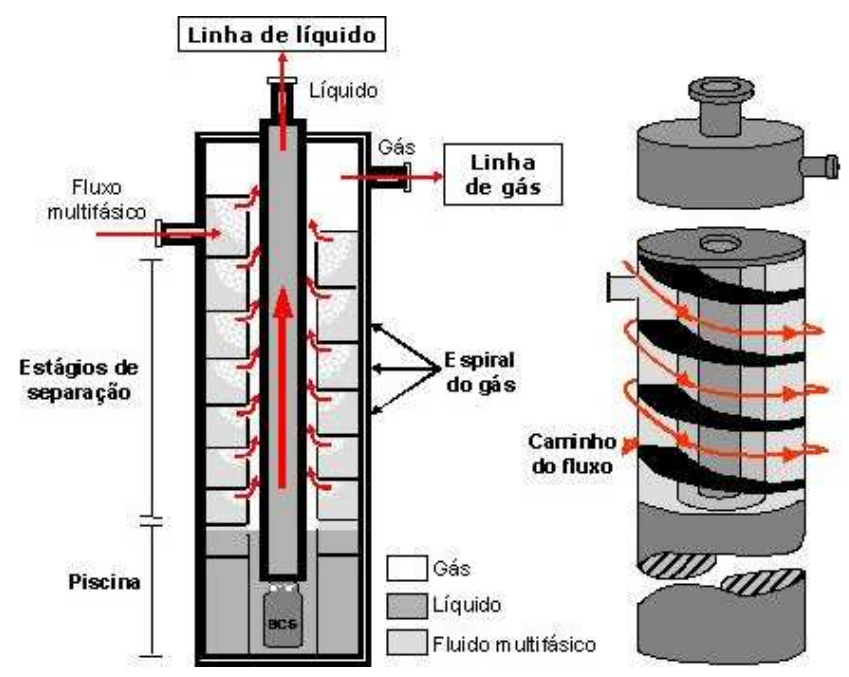

Figura 1: Diagrama esquemático do sistema VASPS.

- Separação terciária: é composta por uma "piscina" na qual o líquido se acumula e parte do gás é separada de forma gravitacional. Nesta piscina opera uma bomba elétrica do tipo BCS.

Para uma melhor compreensão, a Figura 1 apresenta um esquema do sistema VASPS com seus estágios de separação. As setas na figura representam o escoamento de fluido interno ao vaso.

O bocal de entrada dá acesso a uma câmara de expansão que permite que a mistura de líquido e gás descarregue na direção tangencial do separador. O campo centrífugo gerado na saída do bocal propicia que parte do gás seja liberada neste ponto. O líquido e o restante do gás disperso são empurrados contra as paredes, fluindo como um filme em direção a seção da hélice. O filme é acelerado na direção vertical, fluindo para a parte de baixo do separador. A separação gás-líquido ocorre em bolhas dispersas que se movem radialmente para o interior do hélice e alcançam a interface líquido/gás. Sobre o filme flui parte do gás previamente segregado, que alcança o anular de gás através dos furos existentes na parte cilíndrica central da hélice. A piscina na parte inferior do vaso coleta o líquido e algum gás eventualmente disperso. O líquido contido na piscina é bombeado para uma plataforma através da BCS, sendo que esta também atua no controle do nível da piscina. Este controle do nível é necessário porque caso o nível exceda um valor máximo, o líquido pode invadir o espaço ocupado pela hélice e prejudicar o processo de separação. Além disso, um grande aumento de nível pode provocar um aumento na pressão de separação, visto que o nível do líquido reduz o espaço que o gás pode ocupar no separador. No entanto, este aumento de pressão também depende do comprimento da linha de gás até a plataforma de

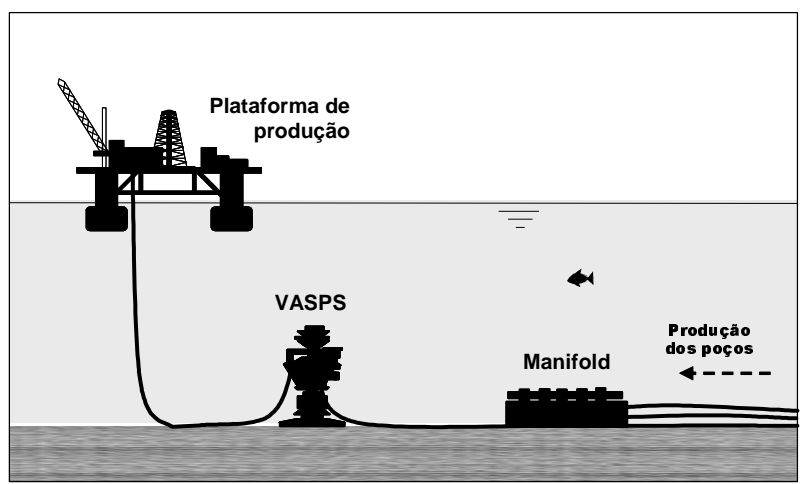

Figura 2: Representação do sistema de produção submarino.

produção. Este comprimento pode alcançar alguns quilômetros fazendo com que a pressão não seja tão sensível ao nível de liquido na piscina. Desta forma, cada caso deve ser analisado com cuidado. Outro ponto, quando o nível está abaixo do mínimo permitido a BCS pode ser danificada.

O sistema VASPS pode ser instalado em um poço falso no leito submarino próximo aos poços de produção. A produção destes poços é escoada para um manifold submarino de produção e, este por sua vez, envia a produção para o VASPS. No VASPS o gás é separado da fase líquida, pelo menos a maior parte. O escoamento após o VASPS é realizado em linhas diferentes de gás e liquido para uma plataforma de produção. O gás escoa para a plataforma pela própria pressão de separação e o líquido é bombeado pela BCS. Uma representação de um sistema de produção com o VASPS está ilustrada na Figura 2.

O sistema VASPS permite reduzir a pressão na cabeça dos poços, proporcionando desta forma um ganho de produção. Além disso, com a separação submarina de gás-liquido evitase vários problemas caracteristicos do escoamento multifásico, como por exemplo, golfadas severas. Outro ponto que merece ser destacado é que, dependendo do cenário, existe a possibilidade de instalar o sistema VASPS em substituição a plataformas que possuem alto custo de manutenção, sendo a produção direcionada para outras plataformas proximas.

Em despeito ao interesse apresentado pela tecnologia, desde sua primeira implantação em campo no Brasil o sistema apresentou algumas dificuldades em manter condições de operação de projeto, dados os distúrbios causados pelo de entrada. Tais distúrbios aliados às não-linearidades de sistema dificultam a análise de tendência pela sua resposta, o que cria a necessidade de acompanhamento próximo de um operador.

Dado tal panorama, surgiu a necessidade de desenvolver um controlador, de forma a melhorar o desempenho do sistema, em contraste com o requisito de preservar o BCS. Os sinais de controle gerados pelo controlador visam solicitar a bomba 
o mínimo possível de modo a evitar o seu desgaste e falhas prematuras.

Para o modelo em utilização neste trabalho, tem-se como variável controlada o nível do reservatório inferior de líquido, apresentado na Figura 1 como 'piscina', e como variável manipulada a frequiência de rotação da bomba, o sinal de controle. A variável controlada deve seguir o valor da referência dentro de certa faixa de estabilidade definida entre um limite inferior (mínimo) e um limite superior (máximo), cujo nível máximo admitido na piscina representa o limiar para o abaixamento da eficiência de separação de bolhas no helicóide, o que alimentaria a bomba com uma fração de gás muito alta no fluido, e cujo nível mínimo na piscina é determinado pela altura física da admissão da bomba, que não deve receber gás já separado. Em ambos os casos, o bombeamento estará comprometido, causando parada no sistema. Portanto, o sistema de controle deve impedir que o nível ultrapasse esses limiares. A solução aqui proposta é voltada para a estabilização do sinal de controle, de forma minimizar a quantidade de rampas de aceleração da bomba que realiza o BCS, aumentando sua vida útil e reduzindo custos operacionais

\section{METODOLOGIA}

O controle voltado ao desempenho de sistemas tem o objetivo de acompanhar o sinal de referência (set point), de forma que a saída do sistema oscile o mínimo possível (Figura 3a). Esta abordagem leva à possibilidade de análise do sinal de resposta para determinar comportamentos assintóticos da resposta do sistema. O sinal de controle, por sua vez, oscila de forma a corrigir os distúrbios impostos no processo, possuindo uma forma muitas vezes similar à do distúrbio de entrada. Este tipo de abordagem é necessário quando a trajetória do sinal de saída é importante, ou quando os limites de estabilidade da saída do processo são muito rígidos. Esta é a técnica mais utilizada na indústria, e já possui metodologias bem estabelecidas e vários produtos comercialmente disponíveis.

Tal metodologia foi testada no VASPS, utilizando controle nebuloso direto (Teixeira et al., 2004, 2006) e controle PID nebuloso (De Melo et al., 2007a-b). Em ambos os casos os controladores apresentam um desempenho satisfatório quanto à redução de sobressinal e tempo de estabilização, apresentando um baixo erro estacionário no regime transiente. Entretanto, a energia de controle é ainda acima do desejável no aspecto referente à necessidade de diminuição da quantidade de acionamentos do atuador.

Em face aos problemas onde o componente crítico do sistema é o atuador, normalmente devido ao seu elevado custo - que pode também ser traduzido como baixo tempo médio entre falhas (MTBF) - é desejável que este permaneça com o mínimo de utilização possível, ou com o mínimo de variações em sua condição de trabalho possíveis, permanecendo no ponto ótimo de operação. Isso acontece no sistema VASPS, onde o motor elétrico da BCS é acionado por um inversor que permite maior flexibilidade através do uso de uma gama de rotações diferentes, mas que acarreta em trabalho fora do ponto ótimo.

Visando a redução do esforço ao qual a bomba está submetida, foi desenvolvido um controlador especificamente voltado ao tratamento do sinal de controle, de forma que este permaneça com poucas alterações, e a saída do sistema (nível da piscina) oscile dentro de uma faixa de estabilidade prevista para o modelo dinâmico do processo, mantendo a eficiência da separação (limite superior) e preservando a bomba (limite inferior). Assim, o comportamento (meramente ilustrativo) desejado para o sistema controlado é apresentado na Figura 3b, e será o objetivo de controle usado no projeto do presente controlador.

No VASPS a redução do esforço de controle pode prover um menor custo ao processo, uma vez que o atuador é o componente crítico, em detrimento do melhor desempenho possível para o sistema. Este compromisso entre desempenho e esforço de controle pode ser parametrizado de acordo com a faixa de estabilidade do processo físico controlado, respeitando seus limites operacionais e utilizando a resposta do sistema para avaliar parâmetros que indiquem quando a alteração no sinal de controle é realmente necessária. Para realizar este tipo de controle, será introduzido aqui o conceito de controle com histeres por auto-ajuste.

\subsection{Modelagem do sistema VASPS}

O sistema VASPS tem se apresentado um eficiente modelo de vaso separador com o método de elevação artificial adaptado à explotação de petróleo em grandes lâminas d'água (Do Vale et al., 2002).

Algumas caracteristicas próprias do VASPS é que ele não é um sistema compacto, possuindo em torno de 70 metros de altura. Também não apresenta uma válvula de controle de fluxo de saída, mas sim uma válvula choke instalada na linha de gás que pode estar alguns quilômetros de distância. Deste modo, é impraticável realizar o controle de nível de líquido na piscina através de uma contrapressão gerada pelo fechamento desta válvula na linha gás. Tampouco se pode realizar controle de pressão no vaso separador com a mesma velocidade (ou mesma 'constante de tempo') do controle de nível de líquido. Por tais razões, neste trabalho foi realizado apenas o controle de nível, tomando-se como restrições tanto o nível alto de líquido, de forma a garantir a eficiência de separação no helicóide, quanto o baixo nível de líquido, de forma a evitar a admissão do gás na bomba, que deve permanecer 


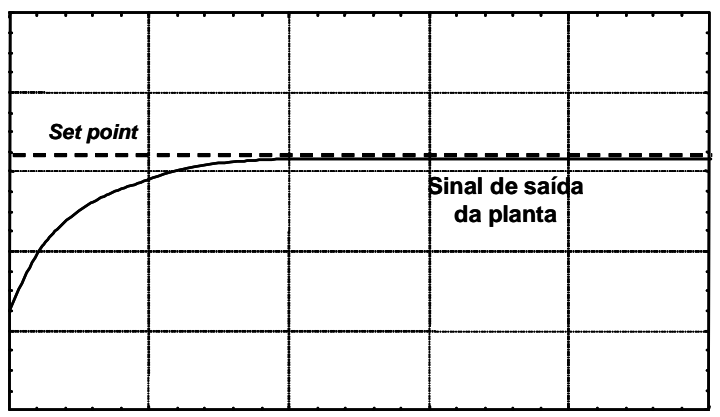

a) controle por estabilidade do sinal de saída

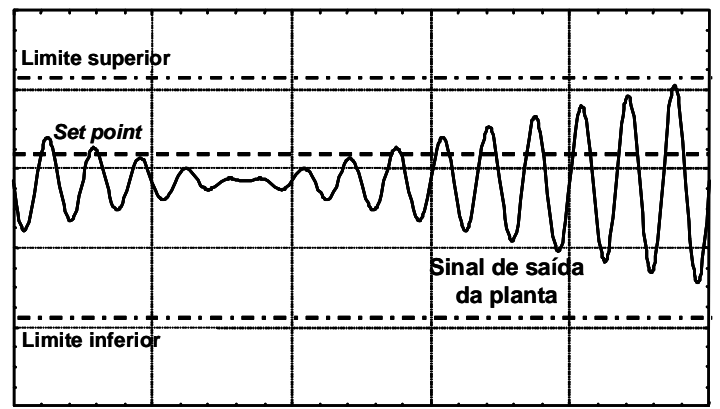

b) controle por minimização do esforço do atuador

Figura 3: Estratégias de controle.

submersa.

No caso de uma linha de gás muito extensa, levou-se em conta apenas a dinâmica da variação do nível de líquido na piscina do separador. Assim, a saída de gás altera a pressão no interno do VASPS, a uma taxa muito menor do que a variação de nível $(h)$ ocorre frente às variações das vazões de entrada $\left(q_{L \_ \text {in }}\right)$ e saída $\left(q_{L_{\_} \text {out }}\right)$. Com isso, pôde-se considerar uma dinâmica mais simplificada com uma a taxa de variação de volume de líquido sendo dada por (1). A variação do nível de líquido foi relacionada à variação de volume $\left(V_{L}\right)$ através de um fator geométrico expresso em (2):

$$
\begin{gathered}
\frac{d V_{L}}{d t}=q_{L \_ \text {in }}-q_{L \_ \text {out }} \\
\frac{d h}{d t}=\frac{1}{\frac{\pi d^{2}}{4}} \frac{d V_{L}}{d t}
\end{gathered}
$$

onde $d$ é o diâmetro interno equivalente do vaso separador na região de presença de líquido.

Como a linha de gás do VASPS até a plataforma é muito extensa, apenas a dinâmica da variação do nível de líquido na piscina do separador foi considerada porque o longo comprimento da linha faz com que o gás não se comprima o suficiente para alterar a pressão de forma significativa, visto que a válvula da linha de gás fica completamente aberta.

Os efeitos complexos da hidrodinâmica do escoamento helicoidal interno ao VASPS não foram considerados uma vez que o escopo deste trabalho foi mais um estudo geral do sistema de controle para, numa segunda etapa, melhorar a modelagem física do sistema.

Sobre à vazão de saída, é necessário considerar outro importante elemento que deve estar presente na malha de controle - o atuador. Inicialmente, considerando a curva de carga do sistema versus a curva da bomba, poder-se-ia imaginar que a vazão constante do sistema no ponto de equilíbrio seria o resultado desta instalação. No entanto, no sistema real, tão embora a carga seja razoavelmente previsível - dada a coluna hidráulica que corriqueiramente é o elemento dominante nesta composição - o acionamento do BCS através de inversor nos permite manipular a curva da bomba através da alteração de sua frequiência de rotação. Com isso, não só a vazão do sistema é alterada, como também a curva de carga sofre variações, uma vez que a componente de perda de carga por escoamento é afetada quando da variação da velocidade do fluido. Assim, o sistema é completamente dinâmico, não se restringindo à avaliação cartesiana de curvas de carga e da bomba estáticas.

O diagrama de blocos do sistema VASPS é apresentado na Figura 4, sendo a curva da bomba BCS não linear expressa de acordo com (3), conforme proposto por Teixeira et al. (2006):

$$
\dot{Q}_{o s}=-0.0577 \cdot f^{3}+8.0786 \cdot f^{2}-249.18 \cdot f-263.36
$$

onde $\boldsymbol{Q}_{o s}$ é a vazão de saída (bomba) em metros cúbicos por segundo e $f$ é a freqüência de alimentação da bomba em Hertz.

Nesta etapa de estudo foi adotada uma abordagem mais simplificada da curva da bomba e desconsiderou-se o efeito da pressão na vazão de saída da bomba. Em trabalhos futuros, esta influência será incorporada ao modelo.

\subsection{Modelagem do distúrbio}

Com o intuito de analisar tanto a robustez do controlador proposto, quanto a sensibilidade do sistema a variações no escoamento, este trabalho apresenta um simulador de fluxo de entrada agressivo. Tal simulador é aqui chamado de 'Gerador de Golfada'. O papel do gerador de golfadas no sistema é importante para a concepção e validação do modelo computacional.

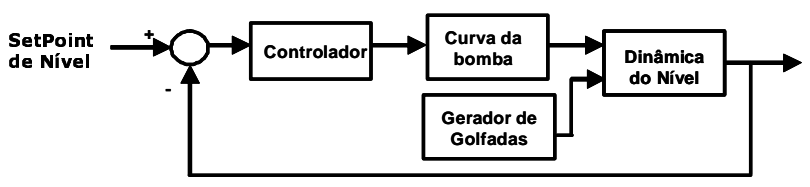

Figura 4: Diagrama de blocos com o detalhamento da planta do sistema VASPS. 


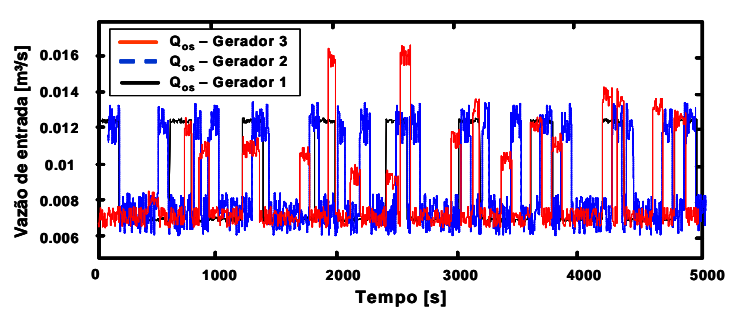

Figura 5: Gerador de golfada.

O gerador de golfadas consiste de uma componente aleatória nos patamares de amplitude da vazão de entrada, assemelhando-se a uma onda quadrada, modificada com a inclusão de três alterações:

1. o aumento da oscilação aleatória no patamar de vazão;

2. a variação da freqüência das golfadas, tornando-as imprevisíveis; e

3. a variação dos patamares de líquido, tornando as golfadas diferentemente agressivas.

O primeiro efeito, representado na Figura 5 como Gerador 1 , foi combinado com o segundo para compor o sinal representado como Gerador 2. A adição do terceiro efeito no gerador permitiu constituir o modelo final para a vazão de entrada, de forma que suas curvas tomam as formas apresentadas na figura como Gerador 3. Este último distúrbio foi o considerado nas simulações do presente trabalho.

\subsection{Modelagem do controlador}

Em trabalhos anteriores, alguns modelos de controladores foram implementados para resolver o problema proposto. Entretanto, estes modelos não se mostraram completamente satisfatórios. O primeiro modelo adotava o conceito de Média Móvel do nível para decidir qual o valor da freqüência ideal. Tal conceito será explicado mais adiante. Este controlador usava um controle nebuloso cujas entradas eram o nível instantâneo e a média do nível (De Melo et al., 2007d). Pode-se observar que neste controle haviam amplas regiões de estabilidade para o sinal de controle e a transição da condição estável para a condição crítica acontecia de maneira suave. Entretanto, estava presente um certo sinal de controle oscilatório entre as os patamares críticos. Além disso, não era possível ter uma variação ampla do set point e havia uma limitação operacional natural na escolha do sinal controle.

Por estas razões propôs-se um controle Classificador baseado na classificação do nível, pela sua proximidade com as situações críticas ou da faixa permitida (De Melo et al., 2007d), onde um controlador nebuloso identificaria o aumento, a diminuição ou a manutenção da freqüência, de acordo com a classificação do sinal de entrada (nível). Este modelo também será explicado posteriormente. Em relação ao desempenho, este controlador permitia a variação do set point, o sinal de controle era mais sensível a alterações do nível, de modo que as faixas de estabilidade tornaram-se mais estreitas. Por outro lado, o nível controlado mostrou não acompanhar as variações no set point e o sinal de controle também continuou oscilatório entre as os patamares de saída.

Nesse contexto, foi proposto um conceito de controlador que mescla ambos os conceitos apresentados anteriormente (Classificador com Média), de forma a reduzir o efeito de grandes distúrbios através da realização de uma média móvel sobre a pertinência classificada, como também limitar a faixa de estabilidade em regiões de trabalho que permitam acompanhar a ordem de grandeza do sinal de referência, ainda permitindo oscilação, mas em torno de mais pontos. Adicionalmente, uma vez que o controlador proposto é mais refinado, sendo também mais sensível às mudanças bruscas do sinal de controle, foi nec faz-se necessário adicionar um filtro de sinal entre o controlador e o atuador, simulando os efeitos de saturação de sinal, quantização e rampa máxima de acionamento.

Este controlador apresentou resultados mais próximo do desejado, com melhorias consideráveis na qualidade do sinal de controle, que mostrou-se mais sensível a alteraçõe, com amplas regiões de estabilidade e transição suave da condição crítica para a estável. Também foi possível variar o set point, mantendo o sinal de controle estável próximo à referência. Contudo, o sinal de controle continuou oscilatório entre as os patamares de saída e houve o estacionamento do sinal de controle entre as regiões de trabalho.

Ainda com as melhorias apresentadas no modelo Classificador com Média, a transição entre conjuntos nebulosos apresentou a dificuldade de se manter na nova região de trabalho até que a correção fosse plenamente estabelecida, o que levava a imaginar a aplicação do conceito de histerese para fixar a variação da saída até que o sistema retornasse completamente à região de trabalho anterior por efeito do próprio comportamento do processo, e não apenas por correção do controlador.

Assim, a histerese é inserida logo após o cálculo de média da pertinência do nível, de forma que o diagrama de blocos do do novo controlador é mostrado na Figura 6.

Com o intuito de uma melhor manipulação da região de trabalho, limitada acima e abaixo, este modelo trata o sinal de entrada com uma função de classificação que retorna uma pertinência relativa à distância entre o limite de estabilidade mais próximo (superior $(L S)$ ou inferior $(L I)$ ) e o valor da 


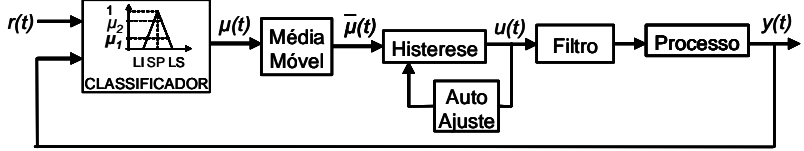

Figura 6: Diagrama de blocos do controlador histerese com auto-ajuste.
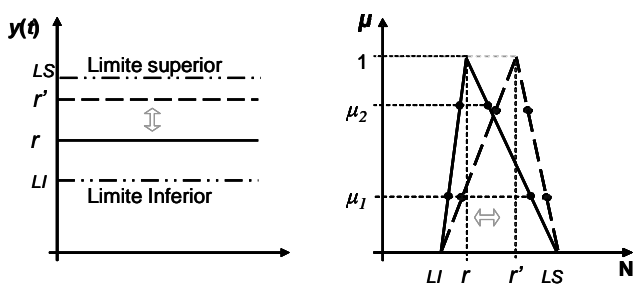

Figura 7: Ilustração da função utilizada pelo classificador.

referência do nível $\boldsymbol{r}(\boldsymbol{t})$. Esta abordagem permite uma livre variação do sinal de referência (set point), dentro da região de trabalho do controlador, de forma que as funções de classificação tomam novos coeficientes, que corrigem a distância entre os limites de estabilidade para o valor da nova referência $\boldsymbol{r}^{\prime}(t)$. A Figura 7 ilustra a construção das funções de classificação de acordo com a referência, e a correção desta após a variação do set point ao longo do processo.

Este conceito, baseado em conjuntos nebulosos (Pedrycz e Gomide, 1998), permite a parametrização da região de trabalho, visto que a entrada é normalizada no intervalo [-1,1]. Com a entrada normalizada, pode-se utilizar a idéia de valor de pertinência $(\mu)$ para esta, dada por (4), de forma que sua magnitude expresse a proximidade à instabilidade ou à referência. Além disso, a inclinação da função de classificação provê uma rápida transição desta pertinência entre estes dois casos:

$$
\mu(t)=\left\{\begin{array}{l}
\frac{y(t)-L I}{r(t)-L I}, y(t)<r(t) \\
\frac{y(t)-L S}{L S-r(t)}, y(t) \geq r(t)
\end{array}\right.
$$

Posto isto, é possível resolver o problema de minimização das variações no acionamento através da atuação restrita à proximidade da região crítica. Com isso em mente, é possível utilizar o classificador para monitorar os limites de estabilidade, e utilizar um mecanismo ajuste da freqüência da bomba, para calcular a rotação nas proximidades da região crítica, realizando correções mais efetivas. No caso do VASPS, o limite inferior $(L I)$ é considerado o nível mínimo admissível para evitar a quebra da bomba $(\boldsymbol{Q B})$ e o limite superior $(L S)$ é o nível máximo admitido para evitar o prejuízo na eficiência da separação de bolhas no helicóide $(\boldsymbol{P S})$.
Adicionalmente, com o objetivo de avaliar a tendência do comportamento do sinal, este controlador realiza uma média móvel de acordo com uma janela parametrizável para cada processo através da variável $n$, de forma que a pertinência média $(\bar{\mu})$ do nível, computado em (5), faz o papel de ajustar o tempo $(t)$ de reação do sistema às variações no sinal de saída $(\mu)$.

$$
\bar{\mu}=\frac{1}{n} \sum_{i=t-n}^{t} \mu(t)
$$

O ajuste realizado por este dispositivo, dependendo do valor selecionado para o parâmetro $n$, pode atrasar uma resposta necessária a um pico de vazão causado por uma golfada na entrada de fluido, e por isso é necessária a manutenção da leitura do nível instantâneo. Uma explicação mais detalhada das abordagens do modelo classificador e da média móvel para o problema de controle pode ser encontrada em De Melo et al. $(2007 \mathrm{c}-\mathrm{d})$.

O comportamento da histerese é dado pelas equações (6), (7), (8), (9) e (10), de forma que as regiões de trabalho podem ser identificadas na Figura 9. Os diferentes intervalos ilustrados na figura correspondem às regiões de trabalho que apresentam um valor constante de saída, fixando o sinal de controle (frequiência de rotação da bomba) enquanto o sistema permanecer na respectiva faixa de trabalho. A alternância entre as equações apresentadas depende das variações na variável avaliada - a pertinência média - cujo universo de trabalho é normalizado no intervalo $[-1,1]$.

$$
u_{P B}(\bar{\mu})= \begin{cases}u_{P B}, & \bar{\mu}(t) \geq \mu_{2}-\delta_{2} \\ u_{P S}(\bar{\mu}), & \bar{\mu}(t)<\mu_{2}-\delta_{2}\end{cases}
$$

$$
\begin{gathered}
u_{P S}(\bar{\mu})= \begin{cases}u_{P B}(\bar{\mu}), & \bar{\mu}(t) \geq \mu_{2} \\
\mathrm{u}_{\mathrm{PS}}, & \mu_{1}-\delta_{1}<\bar{\mu}(t)<\mu_{2} \\
u_{Z}(\bar{\mu}), & \bar{\mu}(t) \leq \mu_{1}-\delta_{1}\end{cases} \\
u_{Z}(\bar{\mu})= \begin{cases}u_{P S}(\bar{\mu}), & \bar{\mu}(t) \geq \mu_{1} \\
\mathrm{u}_{\mathrm{Z}}, & -\mu_{1}<\bar{\mu}(t)<\mu_{1} \\
u_{N S}(\bar{\mu}), & \bar{\mu}(t) \leq-\mu_{1}\end{cases} \\
u_{N S}(\bar{\mu})= \begin{cases}u_{Z}(\bar{\mu}), & \bar{\mu}(t) \geq-\mu_{1}+\delta_{1} \\
\mathrm{u}_{\mathrm{NS}}, & -\mu_{2}<\bar{\mu}(t)<-\mu_{1}+\delta_{1} \\
u_{N B}(\bar{\mu}), & \bar{\mu}(t) \leq-\mu_{2}\end{cases} \\
u_{N B}(\bar{\mu})= \begin{cases}\mathrm{u}_{\mathrm{NB}}, & \bar{\mu}(t)<-\mu_{2}+\delta_{2} \\
u_{N S}(\bar{\mu}), & \bar{\mu}(t) \geq-\mu_{2}+\delta_{2}\end{cases}
\end{gathered}
$$


A associação das funções $u_{N B}(\bar{\mu}), u_{N S}(\bar{\mu}), u_{Z}(\bar{\mu}), u_{P S}(\bar{\mu})$ e $u_{P B}(\bar{\mu})$ constitui a função aplicada ao sinal de controle para gerar seu valor de saída. Este valor de saída por sua vez é dado pelas constantes $\mathbf{u}_{N B}, \mathbf{u}_{N S}, \mathbf{u}_{Z}, \mathbf{u}_{P S}$ e $\mathbf{u}_{P B}$, dependendo do intervalo onde a pertinência média estiver localizada e dependendo também do sinal de derivada de seu valor absoluto, que indica se o valor está se afastando ou se aproximando de zero.

Por sua vez, os valores $\mu_{1}, \mu_{2}$ e seus opostos $-\mu_{1}$ e $-\mu_{2}$ são os valores de transição entre regiões de trabalho quando a derivada do módulo da pertinência média for positiva. Esta condição é chamada de histerese de subida. Por outro lado, caso o módulo da pertinência média estiver decrescendo, ou seja, caso a derivada de seu valor absoluto for negativa, temse a condição de histerese de descida, e neste caso o valor de transição entre as regiões de trabalho é deslocado em direção ao zero de um valor $\delta$, chamado de intervalo de histerese. Assim, pode-se concluir que a transição entre regiões de operação na condição de histerese de descida é dada pelos valores $\left(\mu_{1}-\delta_{1}\right),\left(\mu_{2}-\delta_{2}\right)$ e seus valores opostos $\left(-\mu_{1}+\right.$ $\left.\delta_{1}\right)$ e $\left(-\mu_{2}+\delta_{2}\right)$. Obviamente, como as pertinências usadas para os conjuntos nebulosos de entrada variam no intervalo [-1,1], o universo de trabalho da histerese também está compreendido neste intervalo normalizado.

A Figura 8 ilustra os conceitos de histerese de subida e de descida, bem como as regiões de trabalho rotuladas através do valor da respectiva constante de saída. Nesta, é possível verificar um curioso efeito da histerese sobre as regiões de trabalho, a saber:

- Quando a região está ativa, o valor de transição para as regiões vizinhas é dado pelo maior delta dentre os valores de transição em seu entorno. Assim, pode-se dizer que o intervalo de trabalho é dado pela região expandida no entorno do valor médio da pertinência para aquela região.

- Quando a região não está ativa, para fins de transição de regiões, ela se afasta do zero de um valor igual ao intervalo de histerese. Dessa forma pode-se dizer que o intervalo de trabalho inativo total é dado pela região retraída fora do entorno do valor médio da pertinência.

Este efeito nos permite verificar que a soma das regiões de trabalho ativas é maior do que o intervalo de operação total de um valor igual à soma de todos os intervalos de histerese encontrados entre as fronteiras de operação. Esta maior região de trabalho real permite reduzir significativamente os acionamentos do atuador, zelando pela sua vida útil. Dessa forma, a Figura 8 ilustra a região expandida no entorno da pertinência média igual a zero. $\mathrm{O}$ valor da saída da histerese

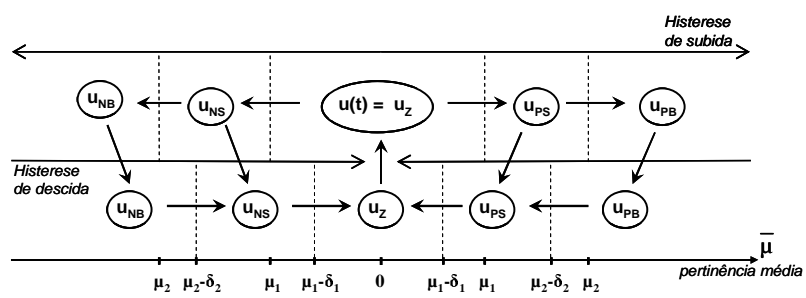

Figura 8: Esquemático ilustrativo do comportamento da histerese.

$u(t)$, como dado no diagrama de blocos, é dado pela função $u_{Z}(\bar{\mu})$, cujo valor de saída é $\mathbf{u}_{Z}$.

Neste ponto, pode-se retomar uma das considerações desenvolvidas na modelagem física do sistema VASPS, a dinâmica da variação da pressão. Havia-se considerado que o tempo de resposta da variação de pressão de gás era muito maior do que o do sistema de controle de nível, de forma que trabalhando no transiente do controle de nível o sistema não seria afetado pela dinâmica da pressão. Assim, embora os efeitos da variação da pressão não estejam claros na malha de controle de nível, se for tomado um tempo muito grande de avaliação da dinâmica interna do VASPS, talvez a amplitude de variação da pressão influencie a tal ponto que os valores constantes de saída $\mathbf{u}_{N B}, \mathbf{u}_{N S}, \mathbf{u}_{Z}, \mathbf{u}_{P S}$ e $\mathbf{u}_{P B}$ não mais estejam adequados às regiões de trabalho definidas para estes. Ou ainda, considerando tempos maiores, a depleção do reservatório pode implicar na alimentação do sistema VASPS com uma pressão de admissão - ou uma vazão de líquido declinante, causando o mesmo efeito.

Assim, considerando-se o problema de minimização de esforço de controle, e na tentativa de adaptar o controlador a condições dinâmicas de operação do sistema ao longo do tempo, foi desenvolvido um mecanismo de correção do valor do sinal de controle no equilíbrio, $\mathbf{u}_{Z}$, de forma que caso o sistema opere certa proporção de seu tempo fora da região de pertinência média igual a zero, o valor de $\mathbf{u}_{Z}$ seja atualizado proporcionalmente. A importância desta correção é ilustrada por observações realizadas em simulação para um sistema dinamicamente variante no tempo, onde a faixa de erro nulo aplicando o sinal de controle $u(t)$ invariante no valor de $\mathbf{u}_{Z}$, gerou atuações que não mantiveram o sistema em equilíbrio, sendo necessária a atuação da faixa de operação vizinha para corrigir o sinal de saída $y(t)$ e retornar a minimizar o erro.

Para realizar o auto-ajuste, o regulador utiliza a média móvel do sinal de controle e a monitora até que esta atinja um valor entre $\mathbf{u}_{Z}$ e $\mathbf{u}_{P S}$ - por exemplo, $70 \%$ da distância entre os patamares - permanecendo ainda mais próximo do valor de equilíbrio, mas já indicando a tendência do sinal de controle se deslocar em direção a uma das extremidades da região de trabalho. Após este valor da média móvel ser atingido, $\mathbf{u}_{Z}$ 
assume este valor, e o sistema passa a ser referenciado a partir daí, sendo este o novo ponto de equilíbrio.

Tendo sido idealizado para aplicações com uma grande constante de tempo, o controlador também apresenta ferramentas que o permite lidar com sistemas mais rápidos, dado o alto grau de informações que este utiliza para definir a política de controle. A formulação do controlador real pode ser também mais ou menos conservadora, uma vez que os parâmetros do sistema permitem definir os limites permitidos para a variável ontrolada e para a variável manipulada.

O mérito de minimizar o esforço de controle requer, como reforçado no início da discussão, uma flexibilidade operacional dos parâmetros de desempenho. Não obstante, as tentativas de minimizar o erro estacionário foram desenvolvidas de forma a contemplar tanto o sinal de controle, quanto de permitir brechas para que o compromisso com o desempenho fosse deixado para a decisão do projetista, podendo este dar um maior peso ao desempenho.

O filtro de sinal inserido na malha tem o objetivo de simular as mínimas variações que são aplicadas ao sinal de controle antes que este seja aplicado aos terminais elétricos da bomba $\mathrm{BCS}$, corrigindo picos de sinal e ruídos. Basicamente, o filtro é constituído de três elementos, aplicados na seqüência a seguir:

- saturador - este elemento de segurança impede que o acionamento da bomba fique fora do intervalo estipulado como limites de funcionamento físico, limitando o universo de atuação das freqüências do inversor.

- limitador de rampa - este dispositivo limita a derivada do sinal de controle a um valor máximo e a um valor mínimo, simulando as rampas de aceleração $(r l s)$ e desaceleração permitidas no sistema $(f s)$. Este limitador atua na malha de controle elétrico da bomba, que possui uma constante de tempo baixíssima, por vezes da ordem de milissegundos, de forma que as variações neste loop interno da bomba não se fazem perceptíveis no controle de nível. De qualquer forma, acionamentos repentinos na forma de ondas completamente quadradas são evitados.

- quantizador - o último componente do filtro aplica um segurador de ordem zero, ou filtro $\mathrm{ZOH}$, ao acionamento do sinal de controle na bomba, evitando sua oscilação como sinal contínuo e portanto minimizando a oscilação do sinal nos terminais da bomba. Neste projeto adotou-se degraus de $0,5 \mathrm{~Hz}$, de forma a realizar variações suaves no acionamento.

As funções aplicadas ao sinal de controle são dadas, respectivamente em (11), (12) e (13):

$$
u_{S}=f_{S}(u)=\left\{\begin{array}{lr}
\max (u), & u>u_{\max } \\
u, & u_{\min } \leq u \leq u_{\max } \\
\min (u), & u<u_{\min }
\end{array}\right.
$$

$$
\begin{aligned}
& u_{L R}(t)=f_{L R}\left(u_{S}(t)\right)= \\
& \left\{\begin{array}{c}
u_{S}(t-1)+u_{\text {max }}^{\prime}, \quad u_{S}(t)>u_{S}(t-1)+u_{\max }^{\prime} \\
u_{S}(t), u_{S}(t-1)-u_{\min }^{\prime} \leq u_{S}(t) \leq \\
u_{S}(t-1)+u_{\text {max }}^{\prime} \\
u_{S}(t-1)-u_{\text {min }}^{\prime}, \quad u_{S}(t)<\begin{array}{r}
u_{S}(t-1)-u_{\text {min }}^{\prime}
\end{array} \\
\text { onde } u^{\prime} \text { é a derivada de } u_{S}(t) \text { no tempo t. }
\end{array}\right.
\end{aligned}
$$

$$
\begin{aligned}
& u_{Q}(t)=f_{Q}\left(u_{L R}(t)\right)= \\
& \left\{\begin{array}{rr}
u_{L R}(t-1)+0.5, & u_{L R}(t) \geq u_{L R}(t-1)+0.5 \\
u_{L R}(t-1), & u_{L R}(t-1)-0.5< \\
u_{L R}(t-1)-0.5, & u_{L R}(t)<u_{L R}(t-1)+0.5
\end{array}\right. \\
&
\end{aligned}
$$

Com este filtro, caso haja qualquer problema com o cômputo do sinal de controle acionando a BCS indevidamente, o sinal mais crítico a ser aplicado à bomba é um sinal com variação de meio em meio hertz, com derivada limitada e valor absoluto limitado por mínimos e máximos, permanecendo dentro do universo desejado.

\section{RESULTADOS}

Uma vez que este controlador abrange os conceitos de um classificador na entrada e o cálculo da média móvel, é necessário realizar simulações para todo o conjunto de parâmetros. No entanto, serão feitas duas análises separadamente - uma para o controlador em si e sua dinâmica junto ao processo, e outra para a avaliação do efeito do mecanismo de auto-ajuste. Para conseguir uma simulação apenas avaliando o controlador, sem o mecanismo de auto-ajuste, basta manter a entrada do gerador de golfadas do sistema como descrito na seção 2.2, pois o mecanismo de auto-ajuste apenas tem efeito após o sistema sofrer mudanças dinâmicas. No caso do VASPS, essa mudança dinâmica seria a depleção do reservatório de óleo, com o decaimento da pressão na formação. Considerando apenas a simulação do mecanismo de auto-ajuste, pode-se simular uma vazão de entrada decaindo e manter os parâmetros do controlador constantes, de forma a avaliar as correções impostas às freqüências de acionamento, $\mathbf{u}_{Z}, \mathbf{u}_{P S}$ e $\mathbf{u}_{N S}$. 


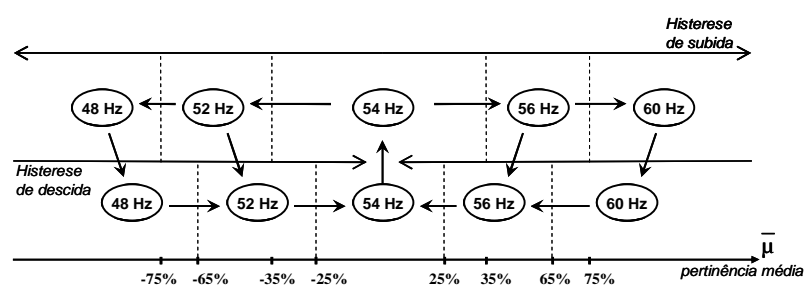

Figura 9: Esquemático da histerese adaptado para o caso VASPS.

\subsection{Avaliação do controlador}

Para a avaliação do controlador foram realizadas inicialmente simulações variando apenas o nível inicial e o sinal de referência, a fim de avaliar o transiente. Os demais parâmetros, como as fronteiras entre regiões de trabalho, $\mu_{1}$ e $\mu_{2}$, a janela de histerese, $\delta$, o valor da saída em cada região, $\mathbf{u}_{N B}$, $\mathbf{u}_{N S}, \mathbf{u}_{Z}, \mathbf{u}_{P S}$ e $\mathbf{u}_{P B}$, os limites de estabilidade do sistema, $\boldsymbol{Q B}$ e $\boldsymbol{P S}$, e o número de pontos utilizados na média móvel, $\boldsymbol{n}$, foram mantidos constantes nas simulações iniciais. A Figura 9 apresenta um esquemático para estas configurações, com os valores de $\mu_{1}$ igual a 0,35 e $\mu_{2}$ igual a $0,75, \delta$ igual a $10 \%, \boldsymbol{u}_{Z}$ valendo $54 \mathrm{~Hz}, \mathbf{u}_{P S}$ valendo $56 \mathrm{~Hz}, \mathbf{u}_{N S}$ em $52 \mathrm{~Hz}$, $\mathbf{u}_{P B}$ em $60 \mathrm{~Hz}$ e $\mathbf{u}_{N B}$ em $48 \mathrm{~Hz}$. Por fim, $\boldsymbol{n}$ foi configurado para 2000 pontos, $\boldsymbol{r l} \boldsymbol{s}$ e $\boldsymbol{f l s}$ para $2 \%$, taxa de amostragem para 0,1 segundo, $\boldsymbol{P S}$ em 20 metros e $\boldsymbol{Q B}$ em 40 metros.

Foram realizadas quatro simulações com estes parâmetros. Para este controlador foi utilizado o gerador de golfadas apresentado, com amplitude e frequiência das golfadas de líquido variáveis. No primeiro par de simulações, mostrados na Figura 10, a referência foi mantida constante (30 metros). O nível inicial $\left(N_{0}\right)$ da piscina no sistema VASPS partiu com 30 metros no gráfico da Figura 10.a, cuja reposta do controle da bomba é apresentada na Figura 10.c. Outra simulação com partida do nível inicial em 60 metros é mostrada na Figura 10.b, com o respectivo resultado do controlador indicado em 10.d.

No segundo par de simulações, apresentados na Figura 11, variou-se o sinal de referência no segundo conjunto de simulações conforme o sinal pontilhado apresentado nas Figuras 11.a e 11.b, onde na primeira figura a partida do VASPS inicia-se com 30 metros, e na segunda com 60 metros. Os respectivos sinais de controle são mostrados nas Figuras 11.c e 11.d.

Este modelo apresentou, pelas simulações, um sinal de controle sem oscilações curtas e freqüentes, bem como um erro estacionário condicionalmente proporcional. No entanto, o grau de compromisso entre qualidade do sinal de controle e desempenho, pode ser manipulado pelos parâmetros que foram mantidos constantes nestas simulações. Além disso, o controlador foi submetido a bruscas variações da referência, não apresentando oscilações indevidas no sinal de controle, e acompanhando a referência dentro de uma faixa de proximidade.

As variações da amplitude do sinal de saída, a rotação da bomba, para esta resposta parecem ser excedentes ao desejado para o VASPS, mas isso ocorre devido à quantidade de variações na referência no curto período de tempo. Caso o operador venha a fazê-lo em campo, o sistema obedecerá, pois está sendo projetado para isso. A recomendação, no entanto, é que caso se deseje a vida útil mais longa possível para a BCS, menores devem ser as variações na referência.

O sistema apresentou uma transição de estados suave ao sair do patamar de nível inicial de 60 metros. A transição foi tão paulatina quanto a variação do nível permitiu. Embora este controlador apresente uma qualidade visível no sinal de controle, as simulações iniciais apresentaram um desempenho que ainda deixa a desejar no tempo de resposta e no erro estacionário, tão embora os resultados se apresentem muito próximo do desejado para o VASPS. As oscilações obviamente não permitem um controle de nível exemplar, mas o controle de rotação, que é o foco deste modelo, apresentase adequado para as simulações de longa duração que foram tomadas a partir de então.

Após diversos experimentos, variando-se os parâmetros do controlador das mais diversas maneiras, as curvas conclusivas do melhor desempenho encontrado são apresentadas na Figura 12, onde se pode notar a melhoria em relação às simulações iniciais. No experimento com o desempenho mais próximo do desejado, a configuração usada considerou os valores de $\mu_{1}$ igual a 0,4 e $\mu_{2}$ igual a 0,$8 ; \delta_{1}$ igual a $40 \%$ e $\delta_{2}$ igual a 30\%; $\boldsymbol{n}$ com 6000 pontos; $\boldsymbol{r l s}$ e $f l s$ em 10\%; taxa de amostragem de 0,1 segundo; $P S$ em 20 metros e $\boldsymbol{Q B}$ em 40 metros. As variações dos limiares de histerese foram investigadas em dois casos, com os mesmos parâmetros acima estabelecidos. No primeiro caso, a variação de um limiar para outro (intervalo de histerese) na região aceitável de trabalho altera em $1 \mathrm{~Hz}$ a freqüência da rotação da bomba e nos casos críticos as frequiências são as rotações mínima e máxima admissíveis da bomba. Assim, tem-se os valores de $\boldsymbol{u}_{Z}=54$ $\mathrm{Hz}, \mathbf{u}_{P S}=55 \mathrm{~Hz}, \mathbf{u}_{N S}=53 \mathrm{~Hz}, \mathbf{u}_{P B}=60 \mathrm{~Hz}$ e $\mathbf{u}_{N B}=48$ $\mathrm{Hz}$. No segundo caso, a variação do limiar nas fronteiras da região de trabalho altera a freqüência de rotação da bomba em 1,5 Hz, de modo que $\boldsymbol{u}_{Z}=54 \mathrm{~Hz}, \mathbf{u}_{P S}=55,5 \mathrm{~Hz}, \mathbf{u}_{N S}$ $=52,5 \mathrm{~Hz}, \mathbf{u}_{P B}=60 \mathrm{~Hz}$ e $\mathbf{u}_{N B}=48 \mathrm{~Hz}$. É importante notar que o tempo de simulação destas curvas são estendidos em relação às simulações anteriores, de forma a realizar uma avaliação mais rigorosa. O tempo de simulação considerado foi de 100.000 unidades de tempo.

É possível notar que após uma avaliação mais profunda do controlador, é possível concluir sobre o sistema a ponto de 
a) Resposta do Nível para o Nível Inicial $\mathbf{N}_{0}=\mathbf{3 0} \mathrm{m}$

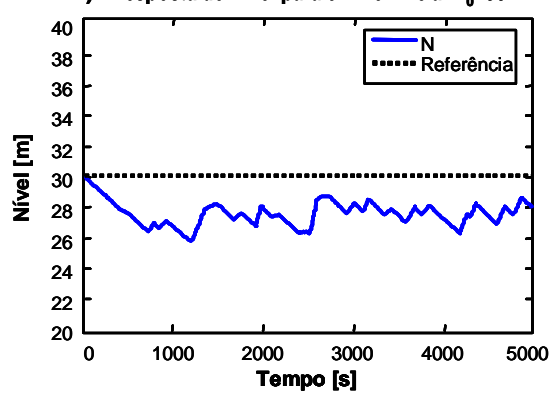

C) Rotação da Bomba para o Nível Inicial $\mathbf{N}_{0}=\mathbf{3 0 ~ m}$

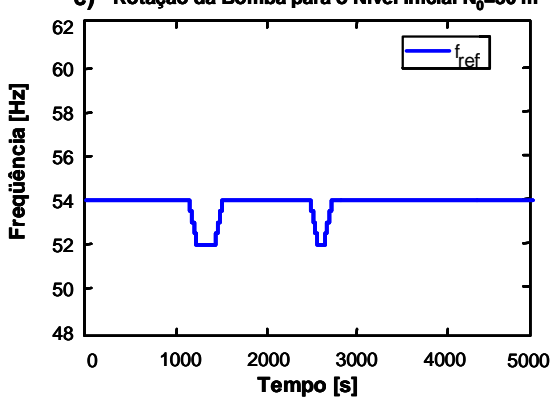

b) Resposta do Nível para o Nível Inicial $\mathrm{N}_{0}=60 \mathrm{~m}$
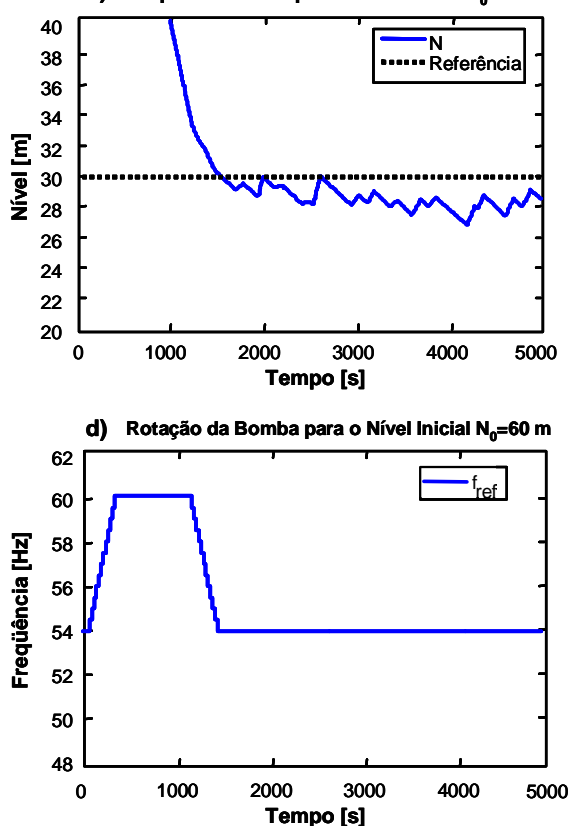

Figura 10: Resposta para o controlador histerese com auto-ajuste, com referência constante.

a) Resposta do Nível para o Nível Inicial $\mathbf{N}_{0}=30 \mathrm{~m}$

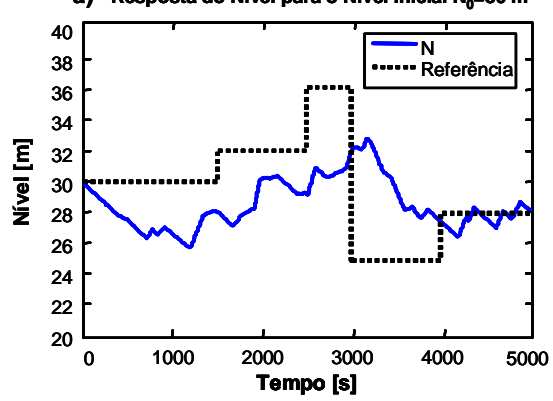

C) Rotação da Bomba para o Nível Inicial $\mathbf{N}_{0}=\mathbf{3 0} \mathrm{m}$

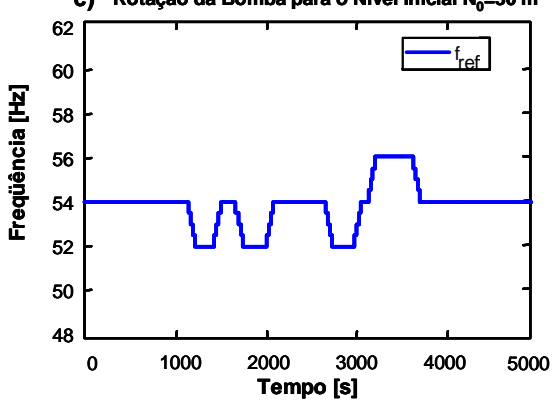

b) Resposta do Nível para o Nível Inicial $\mathrm{N}_{0}=60 \mathrm{~m}$
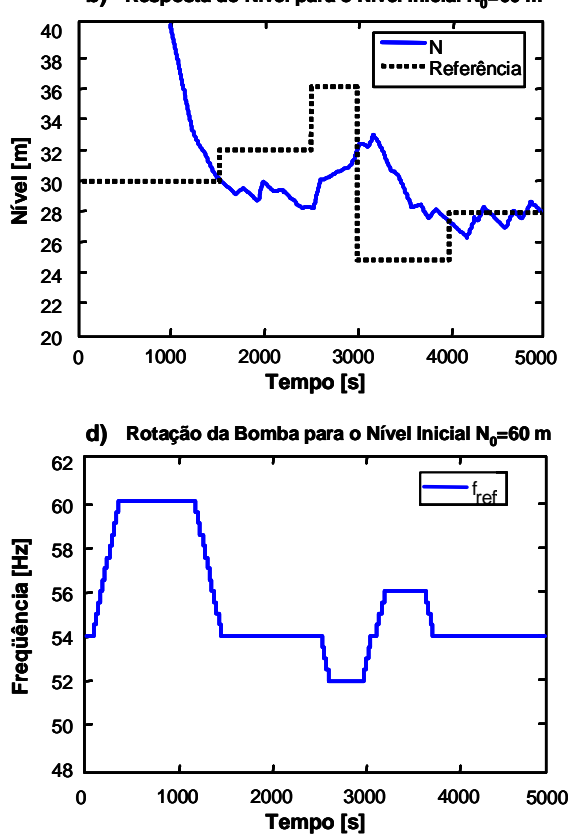

Figura 11: Resposta para o controlador Histerese com Auto-ajuste, com referência variável.

configurá-lo para alguns poucos acionamentos por dia, de forma que mesmo sob as condições mais severas de trabalho, caso o sistema permaneça dentro do escopo de atuação da bomba, é possível corrigir os distúrbios e trabalhar com acionamentos mais demorados e suaves.

\subsection{Avaliação do auto-ajuste}

É possível notar pela Figura 11 que talvez houvesse uma rotação constante para a zona de trabalho próxima ao erro nulo, que permitisse, idealmente, o equilíbrio para um distúrbio conhecido e controlado, dada pela média ponderada das rotações ativadas pelos tempos de atuação decorridos. Essa ro- 

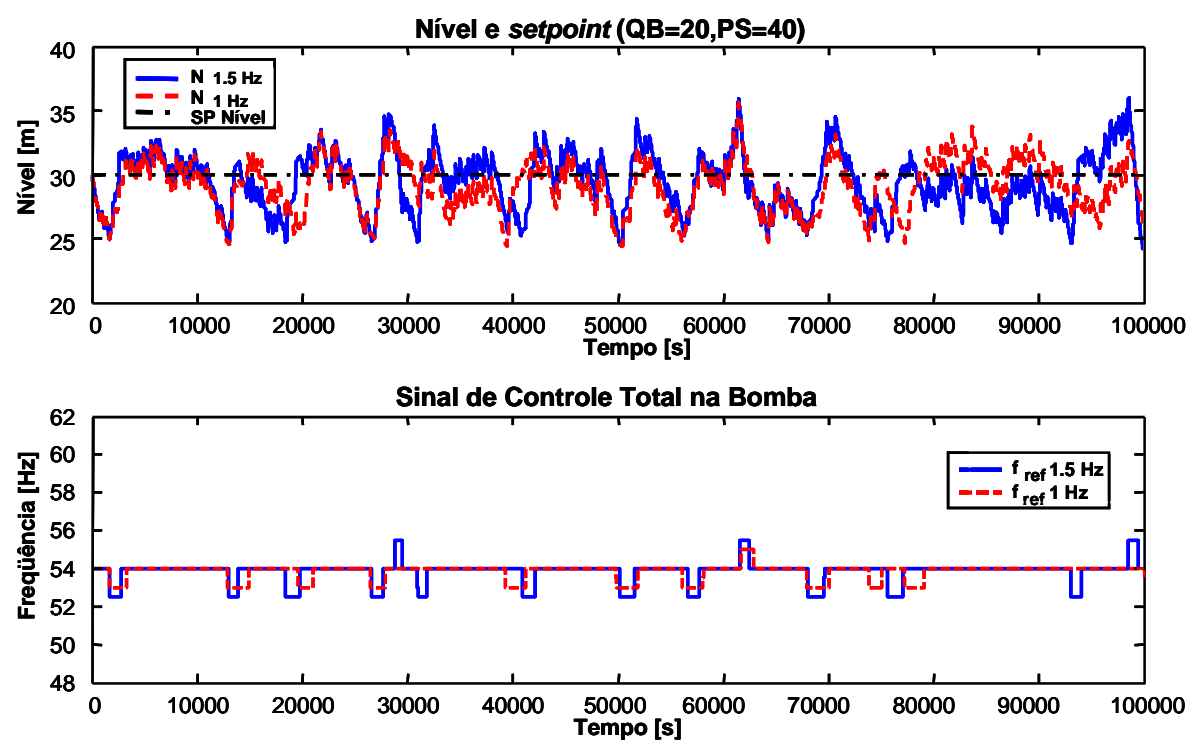

Figura 12: Simulação do controlador com o melhor desempenho.

tação proporcionaria uma vazão de saída que correspondesse ao balanço de massa do sistema. Sabe-se, no entanto, que a vazão recebida pelo VASPS advinda do(s) poço(s) se altera conforme as golfadas evoluem, de forma que o sistema está sendo sempre perturbado, e é impossível manter um equilíbrio perfeito com uma vazão de saída constante.

Conceitualmente, entretanto, é desejado que a vazão de saída no equilíbrio seja o mais próxima possível da média das oscilações da vazão de entrada, de forma a minimizar as alterações no sinal de controle. Assim, considerando que a rotação de saída é ativada em patamares constantes e que oscilam em torno da vazão ideal de saída, é possível computar a média móvel do sinal de controle ativado, da mesma forma que é feito na entrada com a pertinência classificada, e comparálo com os patamares de saída parametrizados, encontrando a distância que a média móvel está de cada um deles. No sistema VASPS, esta distância foi configurada como sendo uma ponderação de $90 \%$ do valor do valor de equilíbrio, $\mathbf{u}_{Z}$, e 10 $\%$ do valor da faixa subsequiente, $\mathbf{u}_{N S}$ ou $\mathbf{u}_{P S}$, de forma que o valor de $\mathbf{u}_{Z}$ é dado em (14):

$$
u_{Z}=\left\{\begin{array}{c}
u_{Z}, \quad\left(0,90 \cdot u_{Z}-0,10 \cdot u_{N S}\right)< \\
\frac{1}{n} \sum_{t-n}^{t} u(t)<\left(0,90 \cdot u_{Z}+0,10 \cdot u_{P S}\right) \\
0.90 \cdot u_{Z}+0.10 \cdot u_{P S}, \\
\frac{1}{n} \sum_{t-n}^{t} u(t) \geq\left(0,90 \cdot u_{Z}+0,10 \cdot u_{P S}\right) \\
0.90 \cdot u_{Z}-0.10 \cdot u_{N S}, \\
\frac{1}{n} \sum_{t-n}^{t} u(t) \leq\left(0,90 \cdot u_{Z}-0,10 \cdot u_{N S}\right)
\end{array}\right.
$$

A arbitragem sobre estes pesos advém das conclusões experimentais que apontaram para a necessidade de mudanças suaves no valor da rotação de equilíbrio, pois quando esta está deslocada do centro da faixa de operação do sinal de controle, mudanças maiores tornam-se muito bruscas no sentido onde há maior margem de variação do nível, a região distante da crítica. Ainda, quando o sistema busca novo patamar de equilíbrio devido a mudanças bruscas no sistema, alterações de maior magnitude no patamar de equilíbrio provaram que o retorno do sistema à condição de erro nulo tem dificuldades em corrigir o valor de $\mathbf{u}_{Z}$ devido à necessidade de uma média de ativações muito alta - ou muito baixa - no sentido oposto do acionamento, o que dificilmente ocorre quando o sistema retorna de um ponto de operação crítico.

Por simplicidade computacional, o valor de saída do sinal de controle na faixa de transição, $\mathbf{u}_{N S}$ ou $\mathbf{u}_{P S}$, é parametrizado pelo valor da saída nos outros intervalos, $\mathbf{u}_{Z}$ e $\mathbf{u}_{N B}$ ou $\mathbf{u}_{P B}$, de forma que ao se alterar o valor de $\mathbf{u}_{Z}$, automaticamente todos os outros patamares para o sinal de controle também sejam corrigidos, de tal forma que o cômputo dos valores de $\mathbf{u}_{N S}$ ou $\mathbf{u}_{P S}$ são indicados em (15) e (16). É interessante notar que um acionamento para $\mathbf{u}_{N S}$ ou $\mathbf{u}_{P S}$ mais distante que a avaliação da média móvel do sinal de controle proporciona às faixas de correção um poder significativo de atuação, permitindo variações na média móvel condizentes com os pesos de $90 \%$ e $10 \%$ propostos.

$$
u_{N S}=\frac{\left(2 \cdot u_{Z}+\cdot u_{N B}\right)}{3}
$$




$$
u_{P S}=\frac{\left(2 \cdot u_{Z}+\cdot u_{P B}\right)}{3}
$$

Finalmente, para realizar as simulações, também é importante ponderar sobre o valor do número de pontos usado na média móvel do sinal de controle. Diferentemente do $\boldsymbol{n}$ utilizado na pertinência de entrada, não se deseja que o parâmetro para o sinal de controle seja muito grande, pois o tempo de reação que o sinal de controle deve possuir sobre o sistema deve ser muito menor, ou seja, sua alteração deve ser mais rápida, que o tempo de reação da resposta, uma vez que é o sinal de controle que propicia uma condição de trabalho estável. Assim, utilizando inicialmente um $\boldsymbol{n}$ para o sinal de controle no valor de 2000 pontos, em vez dos 4000 pontos propostos para o sistema, foram realizadas as simulações cujos resultados foram apresentados na Figura 13.

As curvas apresentam a resposta do nível, o sinal de controle e o valor de $\mathbf{u}_{Z}$ para duas condições simuladas, com valores da vazão de entrada do gerador de golfadas, o distúrbio, dadas pelas Figuras 13.c e 13.d. A primeira condição simula uma transição descontínua de grande amplitude no sistema, como por exemplo se um segundo poço ou manifold tivesse de ser interrompido bruscamente. Há duas transições de forma a melhor avaliar o transiente. A segunda condição representa um declínio linear na magnitude da vazão, representando uma queda linear de pressão no poço. Essa queda seria muito mais suave em campo, uma vez que a simulação possui uma duração de 20000 segundos, o que corresponde a um período de cinco horas e meia de trabalho.

Em ambas as simulações é possível notar pelo valor de $\mathbf{u}_{Z}$ três patamares de trabalho que foram buscados pelo mecanismo de auto-ajuste, bem como o seu transiente, bastante evidente e representado pelas mudanças em degrau, que também foram refletidas no sinal de controle. É interessante notar que embora houvesse ativações das faixas de transição, em momento algum o sistema apresentou instabilidade na alteração de patamares, apresentando o comportamento desejado.

\section{CONCLUSÕES}

O desenvolvimento de sistemas de controle para processos contínuos apresenta possibilidades de melhorias de processo muito relevantes industrialmente. Este trabalho abordou as diferenças conceituais na proposição de métodos de solução dos objetivos de controle, objetivos estes bastante diferentes do caso ordinário encontrado na indústria. Adicionalmente, a aplicação proposta para estes novos conceitos ilustra uma amostra da gama de oportunidades encontradas na indústria do petróleo para o desenvolvimento e aplicação desta tecnologia.
Neste sentido, ao se abordar o problema de campo com uma estratégia de controle diferente da tradicional, focando os esforços no requisito crítico do sistema, a bomba BCS, uma técnica alternativa de controle foi desenvolvida, provendo um controlador cada vez mais robustos frente à agressividade crescente dos parâmetros aplicados nas simulações.

O estudo do caso VASPS apresentou um modelo de controlador voltado para a estabilização do sinal de controle, que mantém o sinal bem comportado, com variações suaves ao longo da região de projeto. Neste controlador, o sinal de controle mostrou-se sensível a alterações de projeto e de parâmetros, permitiu o acompanhamento do sinal com set points variáveis, permitiu manipular a faixa de estabilidade do sistema e possibilitou transições suaves de condições críticas para estáveis.

Embora o controlador desenvolvido não possa ser comparado diretamente com outros tipos de controladores, haja vista que este sistema de separação e bombeamento submarino possui um caráter único e inovador, sem concorrentes industriais diretos, e muito menos apresenta tecnologia própria de controle estabelecida, é possível estimar a eficácia do controlador proposto contrastando com versões anteriores propostas e também através de informações advindas da sua operação do VASPS em campo. Os testes desenvolvidos para o controlador permitiram partir de uma configuração com cerca de 40 acionamentos curtos de velocidades diferentes no sinal de controle com os modelos de controladores precedentes mencionados no curso deste artigo para, com a presente abordagem, algo em torno de apenas 10 acionamentos, considerando um dia de trabalho, o que está condizente com a informação de campo de que cada alteração na rotação da bomba é seguida de um intervalo de 2 a 3 horas no procedimento manual com acompanhamento de um operador. Além disso, outro ponto a considerar é que a duração dos acionamentos apresentaram duração maior que nos estudos anteriores. O sistema aqui simulado, no entanto, utilizou um sinal de distúrbio de entrada mais intenso que a média encontrada em campo, justamente para suplantar a desconfiança inerente a um modelo computacional.

A adoção desta estratégia de controle permitiu o balanceamento do compromisso desempenho versus esforço de controle. Portanto, o objetivo de reduzir o custo da operação em alto mar através da redução no número de intervenções submarinas causada pelo aumento da vida útil da bomba BCS foi plenamente alcançado.

Também é importante frisar que esta técnica de controle inteligente usando histerese com auto-ajuste não só se mostrou adequada para o tratamento de sistemas contínuos, como também permitem uma maior facilidade na tomada de decisão automática para sintonia de parâmetros de controle. 
a) Resposta do sistema - Nível em metros
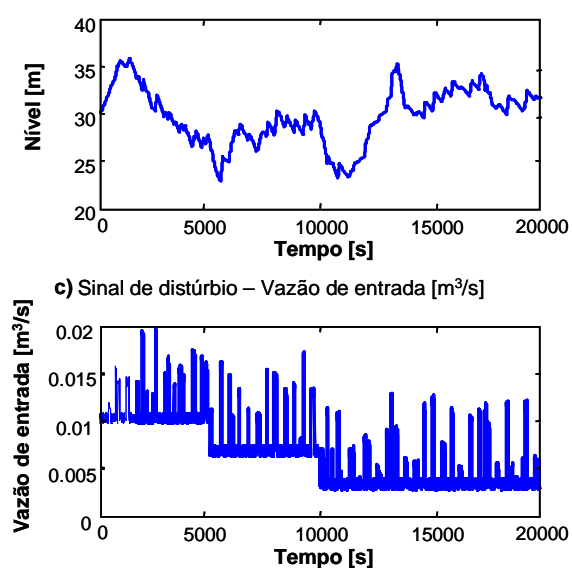

e) Sinais de Controle Total e de Equilíbrio

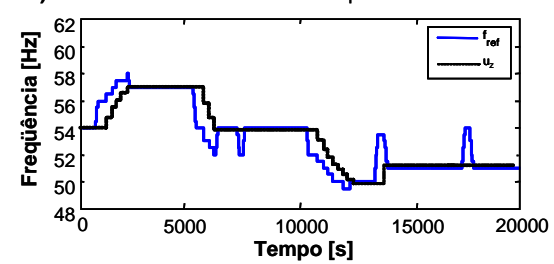

b) Resposta do sistema - Nível em metros
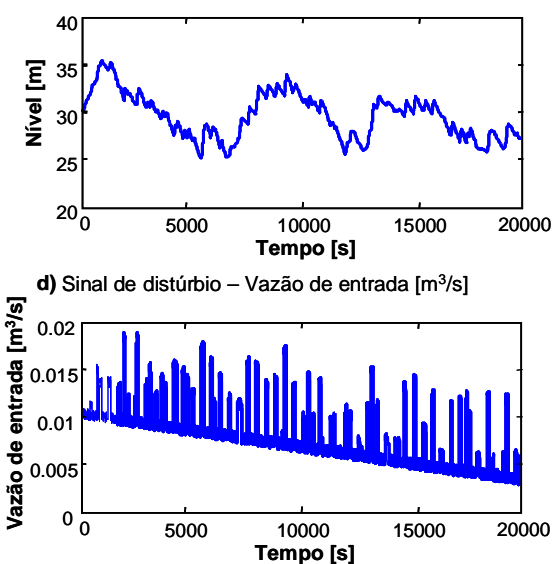

f) Sinais de Controle Total e de Equilíbrio

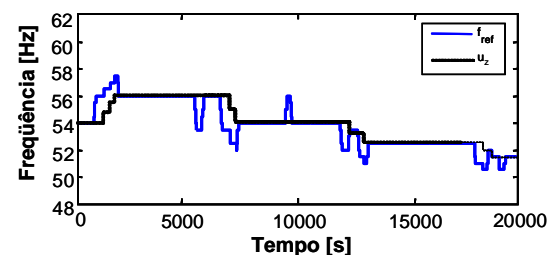

Figura 13: Curvas para as simulações de teste do mecanismo de auto-ajuste.

Neste sentido, há aplicações muito promissoras na área de controle para o desenvolvimento de técnicas de produção submarina, facilitando o gerenciamento remoto de campos de petróleo. É de fundamental interesse para a indústria que a confiabilidade dos sistemas seja alta, e o controle inteligente, por sua forma de concepção, pode prover a flexibilidade necessária.

Para projetos futuros, uma validação do controlador funcionando em uma aparato experimental em escala que reproduz as características do separador submarido deverá ser realizada em breve. Em termos de melhoria de projeto de controle, um novo modelo que introduza também análises preditivas de comportamento mostra-se bem interessante.

\section{REFERÊNCIAS}

De Melo, A.V.; Mendes, J.R.P.; Serapião, A.B.S. and Estevam, V (2007a), Fuzzy tuned PID control for the VASPS subsea separator, 6th Brazilian Conference on Dynamics, Control and their Applications (DINCON), São José do Rio Preto.

De Melo, A.V.; Mendes, J.R.P.; Serapião, A.B.S; Guilherme I.R.. e Estevam, V (2007b), Controle supervisório inteligente para o separador submarino VASPS, Anais do IV Congresso Rio Automação, IBP568_07, Instituto Brasileiro de Petróleo e Gás, Rio de Janeiro.
De Melo, A.V.; Mendes, J.R.P.; Serapião, A.B.S. e Estevam, V (2007c). Minimização de esforço de controle para processos contínuos - estudo do separador VASPS, Revista Petro\&Química, Vol.299.

De Melo, A.V.; Mendes, J.R.P; Serapião, A.B.S. and Estevam, V (2007d). Intelligent Supervision Control for the Vasps Separator, Brazilian Journal of Petroleum and Gas, Vol.1, No.2, pp. 67-77.

Do Vale, O.R.; Garcia, J.E. and Villa, M (2002), VASPS installation and operation at Campos Basin. Offshore Technology Conference, OTC 14003, Houston, Texas.

França, F.A.; Rosa, E.S.; Bannwart, A.C.; Moura, L.F. and Alhanati, F.J (1996), Hydrodynamic studies on a cyclonic separator, Offshore Technology Conference, OTC 8059, Houston, Texas.

Pedrycz, W. and Gomide, F (1998). An Introduction to fuzzy sets: analysis and design, Massachusetts: MIT Press, 465p.

Teixeira, A.F.; Mendes, J.R.P.; Serapião, A.B.S. e Guilherme, I.R (2006). Controle inteligente para o sistema de separação e bombeamento submarino - VASPS, Rio Oil \& Gás Expo and Conference, IBP1802_06, Rio de Janeiro.

Teixeira, A.F.; Mendes, J.R.P.; Estevam, V.; Guilherme, I.R.; Morooka, C.K. e RIGO, J.E (2004), Um controlador 
fuzzy para o sistema de separação e bombeamento submarino - VASPS, Rio Oil \& Gás Expo and Conference, IBP11804, Rio de Janeiro.

Wang, S.; Mohan, R.S.; Shoham, O. and Kouba, G.E (2001), Dynamic Simulation and Control-System Design for Gas/Liquid Cylindrical Cyclone Separators, SPE 71308, SPE Journal, pp.236-247. 